\title{
Lo cuqui va a la guerra: estrategias de comunicación probélicas en videojuegos japoneses
}

\author{
Enrique GALVÁN JEREZ \\ Departamento de Lengua Española y Teoría de la Literatura y Literatura Comparada \\ Universidad Complutense de Madrid \\ enrique.galvanjerez@gmail.com
}

\begin{abstract}
RESUMEN
Al igual que las películas o la literatura, los videojuegos también pueden expresar una visión positiva de la guerra. Por ejemplo, son parte de la propaganda de ejércitos, como el de EE.UU., o de grupos terroristas, como Hizbolá. En el caso de los videojuegos japoneses, estos son interesantes por sus estrategias comunicativas inusuales dentro de un país que fomenta la cultura antibélica. Este artículo investiga cuáles son estas estrategias retóricas en dos videojuegos: Squishy Tank (2010), de Success, y Valkyria Chronicles (2008), de Sega. Para ello, se aplicarán los conceptos estéticos japoneses del kawaii, lo adorable, y el moe, la identificación y el fetichismo con un personaje ficticio.
\end{abstract}

Palabras clave: videojugeos, kawaii, retórica probélica, Squishy Tank, Valkirya Chronicles.

\section{Cuteness Goes to War: Pro-War Strategies of Communication in Japanese Video Games}

\begin{abstract}
The same as literature or films, video games can also express a positive view of the war. For example, they are part of the propaganda of armies, such as the US Army, or terrorist groups, such as Hezbollah. Related to Japanese video games, they are interesting due to their unusual strategic communications within a country which promotes pacifist culture. This paper analyses which are these rhetorical strategies in two video games: Squishy Tank (2010), by Success, and Valkyria Chronicles (2008), by Sega. In order to achieve it, it will employ the Japanese aesthetic concepts of kawaii, cuteness, and moe, the identification and fetishism with a fictional character.
\end{abstract}

Keywords: video games, kawaii, pro-war rhetoric, Squishy Tank, Valkirya Chronicles.

\section{Introducción}

Desde los albores de la humanidad, gobiernos y organizaciones han utilizado distintos métodos para favorecer una visión positiva de la guerra. Uno de estos 
métodos es la concesión de derechos, bienes y favores. Por ejemplo, durante la Antigüedad, la guerra era un método para conseguir la ciudadanía en las ciudades griegas y en Roma. Más adelante, en la Edad Media, era frecuente que la Iglesia católica apoyara los intentos de conquista y expansión del cristianismo con la concesión de bulas, riquezas o derechos políticos.

En la actualidad, este tipo de mensajes continúan. Las promesas de riquezas, aventura y de sexo aparecen en la publicidad de distintos ejércitos y grupos terroristas. No obstante, este tipo de propaganda está dirigida a un segmento de la población específico para su reclutamiento. Por lo tanto, hay sectores, como las mujeres o lo niños, que no reciben este contenido probélico. Para alcanzar este objetivo, utilizan distintas expresiones culturales a su alcance.

Formas cultures más tradicionales están dentro de los instrumentos de publicidad. En la literatura, el escritor Edith Wharton, bajo la petición de Theodore Roosevelt, publicó novelas a favor de la intervención de EE.UU. en la Primera Guerra Mundial (Tate 2009: 179). Dentro del cine, la película Amanecer Rojo (1984), donde narra los esfuerzos de un grupo de jóvenes que luchan contra la invasión del territorio norteamericano por parte de la Unión Soviética y Cuba, aboga por el desarrollo de milicias para evitar una invasión comunista. Incluso la animación es otra de estas herramientas, como el cortometraje de Walt Disney Der Fuehrer's Face (1943), que relata las vivencias oníricas del Pato Donald en una de las potencias del eje. Con esta variedad de medios utilizados, los videojuegos pueden ser una nueva forma de diseminación de este tipo de proclamas.

En la actualidad, Japón no es un caso aislado en la propaganda probélica. Desde la derrota en la Segunda Guerra Mundial y la ocupación americana, las instituciones oficiales promueven los intercambios culturales y los actos relacionados con la consecución de la paz mundial, como el programa «El barco mundial de la juventud» (Sekai Seinen no fune).

Además, la cultura también promueve el pacifismo de distintas formas, como la novela Crónica del pájaro que da cuerda al mundo (1994), de Haruki Murakami; la película La condición humana (1959), de Masaki Kobayashi; o incluso en cómics como Pies descalzos: Una historia de Hiroshima (1973-1974), de Keiji Nakazawa.

Incluso la sociedad civil se preocupa de esta búsqueda. Esto se refleja en los movimientos estudiantiles (zenkyoto) de los años 60 y principios de los 70 , y otros grupos civiles, que buscaban la paz frente a los acuerdos del gobierno por la firma del acuerdo del tratado de San Francisco en la colaboración militar con los EE.UU. ${ }^{1}$

Sin embargo, muchos de los videojuegos japoneses tienen un componente a favor de la guerra. Aunque la memoria de la derrota y las penalidades sufridas durante la Segunda Guerra Mundial no ha desaparecido, deben recurrir a otros

\footnotetext{
${ }^{1}$ No obstante, también cabe la posibilidad de que muchas de las protestas contra el tratado de Seguridad EE.UU.-Japón fueran por la forma antidemocrática de la firma de los acuerdos. El primer ministro Kishi Nobosuke expulsó a los miembros de la oposición cuando el parlamento ratificó la firma del tratado (Kawato, Pekkanen y Yamamoto 2015: 125).
} 
medios para llevar ese mensaje a los jugadores. ¿Cómo son estas estrategias comunicativas a favor de la guerra?

El objetivo de este artículo es analizar la retórica probélica de dos videojuegos modernos: Squishy Tank (2010), de Success, y Valkyria Chronicles (2008), de Sega. Para ello, se hará un análisis a través del kawaii y el moe, dos elementos de la estética japonesa actual.

\section{La guerra y los videojuegos desde la antropología, la sociología y los game studies}

Aunque pueda parecer lo contrario, la antropología establece relaciones estrechas entre los juegos y la guerra. Johan Huizinga analiza que la guerra y los juegos comparten su naturaleza ritual y su rigor por las reglas:

Muchas veces, ambos conceptos parecen confundirse efectivamente. Cualquier lucha vinculada a reglas limitadoras porta ya, por este ordenamiento regulado, los rasgos esenciales del juego, y se muestra como una forma de juego especialmente intensa, enérgica y muy clara. [...] La lucha como función cultural supone siempre unas reglas limitadoras, y exige, en cierto grado, el reconocimiento de su carácter lúdico. (2010: 117-118)

Según esta cita, el juego y la guerra comparten lo agonal. Agonal, que procede del griego antiguo agon, es el elemento de la competición y lucha. Este elemento es fundamental en muchos juegos de distinta índole, ya sea el parchís, el baloncesto o una partida entre dos jugadores al Street Fighter II: The World Warrior (1991), de Capcom. Más adelante, el sociólogo Roger Caillois lo incluye dentro de los fundamentos del juego junto con el alea (la suerte), mimicry (lo mímico), y el ilinx (el vértigo) (1977). Los videojuegos, al fin y al cabo, son juegos en formato electrónico y están sujetos a estos componentes. Por lo tanto, el juego y la guerra comparten similitudes desde el punto de vista de la antropología ${ }^{2}$ y la sociología.

Además, desde tiempos remotos, los juegos son útiles herramienta para la enseñanza de estrategia militar. Algunos ejemplos son el ajedrez, utilizado en la actualidad, o los juegos de estrategia tipo wargame, como se explica en la siguiente cita:

Chess was an ancient wargame developed to train apprentice commanders on the finer points of battlefield operations as they existed before firearms. By the 1700s and 1800 s, chess had already developed into more complex wargames that most gamers today would recognize as miniatures or manual (board) wargames. In the early nineteenth century, this new form of military wargaming, incorporating recently developed statistical tools, was quickly recognized as militarily useful and became quite

${ }^{2}$ No obstante, Huizinga considera que la Guerra no siempre se puede equipara siempre con la guerra, pues esta incluía formas que no corresponde al uso de reglas como la emboscada o el exterminio (2010: 118) o la dificultad difícil ponderar su carácter agonal (2010: 124). 
popular, effective and widespread as a training and planning tool from that point through World War II. (Dunnigan 2016: XXXIII)

Los simuladores de prácticas de vuelos de combate o juegos de estrategia por ordenador es la evolución lógica. De cualquier modo, los videojuegos no destinados a la transmisión y práctica de conocimientos militares también pueden poseer mensajes probélicos. Investigadores adscritos a los game studies, la disciplina que estudia los juegos desde un panorama polifacético, se encargan de averiguarlos.

Los videojuegos, según Ian Bogost, pueden estar asociados con un componente retórico importante. Para ello, utiliza el término de retórica de procesos -o retórica procedural- que determina las reglas de expresión de un videojuego:

Procedurality refers to a way of creating, explaining, or understanding processes. And processes define the way things work: the methods, techniques, and logics that drive the operation of systems, from mechanical systems like engines to organizational systems like high schools to conceptual systems like religious faith. Rhetoric refers to effective and persuasive expression. Procedural rhetoric, then, is a practice of using processes persuasively. (Bogost 2010: 2-3)

Ian Bogost equipara los distintos componentes y procesos con las distintas herramientas retóricas de las palabras o las imágenes. De esta forma, un videojuego utiliza diferentes estrategias para transmitir un mensaje político, en el sentido de que intenta concienciar a la población sobre un tema. Algunos ejemplos no relacionados con la guerra son Cooking Mama, The Unauthorized Peta Edition: Mama kills animals (2008), sobre las condiciones de los pavos en las granjas, o Starway to Tax Heavens (2016), sobre los papeles de Panamá. Como consecuencia, es lógico que los videojuegos puedan contener y difundir una proclama probélica.

Asimismo, los videojuegos están íntimamente ligados con los desarrollos militares. Patrick Crogan señala que los videojuegos y la tecnocultura no existirían sin tres disciplinas desarrolladas con fines militares: la ciencia de la cibernética, diseñada por el matemático Norbert Wiener, el desarrollo de la red de radares y control de terreno del Ejército del aire de los EE.UU. (SAGE o Semi-Automatic Ground Environment) y el SIMNET, una red informática desarrollada para la simulación de ejercicios militares (2011: 2). Para él, sin estos desarrollos, no existirían las interfaces entre ordenador y ser humano, los juegos masivos en línea o el desarrollo de los videojuegos de simulación. En resumen: Crogan establece que los videojuegos y la cibercultura -o tecnocultura- está ampliamente influida por los desarrollos tecnológicos militares.

Según este hilo argumentativo, no es extraño que organizaciones militares utilicen los videojuegos como arma retórica. En el caso del Ejército de los EE.UU., el videojuego America's Army (2002) es una muestra de ello. Su objetivo es reclutar a posibles candidatos mostrando una imagen atractiva tanto por las situaciones de combate como por el curso de adiestramiento. Es decir, a diferencia de otros juegos como el Counter-Strike (1999), de Valve, el jugador comienza con la acción; en cambio, en America's Army, se deben superar una serie de retos y conseguir puntos 
de experiencia para licenciarse y realizar las misiones de combate. En consecuencia, este videojuego no solo muestra una visión seductora para el usuario, sino que intenta emular de forma más fiel la vida de un soldado.

También organizaciones terroristas utilizan los videojuegos como publicidad. Hizbolá desarrolló videojuegos con ánimos de lanzar un mensaje positivo para su organización. Este es el caso de Special Force 2: Tale of the Truthful Pledge (2007). En este videojuego, el participante debe combatir contra las fuerzas invasoras de Israel, siguiendo los acontecimientos de la Guerra del Líbano del 2006. Para convencer a los jugadores, utiliza los mismos recursos que los videojuegos occidentales del género.

A modo de conclusión, los videojuegos pueden ser una herramienta de propaganda muy eficaz. Por un lado, muestra un lado excitante y con gancho de lo que puede ocurrir en la guerra. Por el otro lado, estas sensaciones permite disfrutarlas de una forma segura y facilitar su alistamiento. El caso japonés es diferente, pues se diferencia tanto por la estética como por las mecánicas. En los siguientes apartados, se analizarán los dos ejemplos propuestos con especial atención a estas diferencias.

\section{El kawaii y sus funciones}

Kawaii es una palabra que, a priori, se puede traducir como «adorable», «cuqui», «mono», «lindo», «rico»... Sin embargo, esta traducción no es fiel. En Occidente, lo adorable se refiere a una realidad visual (Morreall 1991: 39), pero en Japón se asocia también a las texturas y a los sonidos (Cheok y Fernando 2012: 301).

No solo estas diferencias recaen en lo sensorial. Para Inuhiko Yamota, el kawaii es la expresión de cuidar y proteger a los seres indefensos. Así lo escribe en la siguiente cita: «Entonces, ¿cómo pueden ser reconocidos como kawaii por los seres humanos? Esto es porque que ellos, visto desde el lado humano, son impotentes e indefensos dentro de la sociedad humana y confirman que necesitan protección de forma constante» ${ }^{3}$ (2015: 86).

Además, la palabra kawaiisō, una palabra derivada, alude a algo digno de compasión. Este último aspecto, olvidado en las lenguas europeas, remite a las características de lo adorable y su vinculación con la biología.

Konrad Lorenz, el fundador de la etología, investigó el comportamiento tanto de animales como de seres humanos en el trato con las crías. En su estudio El comportamiento humano y los animales, concluye que existe una relación entre el tamaño de la cabeza con esta cualidad estética. Una cabeza de tamaño no proporcionado con el resto del cuerpo provoca que los adultos del grupo, sean humanos o animales, se interesen por la criatura para prestar cuidados (Gould 2008: 366). Por consiguiente, se encuentra una relación entre el tamaño de la cabeza y la cantidad de ternura que provoca en el receptor.

Aparte del tamaño de la cabeza, existen otras características relacionadas a esta cualidad. Sharon Kinsella, determina que elementos que componen el kawaii son los

${ }^{3}$ La cita está traducida por el autor del artículo. 
siguientes: la pequeñez, la blandura, el carácter infantil, ser un mamífero, poseer un cuerpo redondo, la ausencia de boca y fosas nasales, la inexistencia de brazos o piernas, no tener un sexo asignado, la mudez, la inseguridad, su indefensión o su lado salvaje (Kinsella 1995: 226). Estas características están presentes en personajes famosos de la cultura popular japonesa como Hello Kitty, de la empresa Sanrio; Pikachu, del videojuego, Pokemon (1996), de Game Freak; o la mascota de la prefectura de Kumamoto: Kumamon.

Asimismo, lo kawaii posee dos características adicionales. La primera es relajar la comunicación por su informalidad. Este uso de una comunicación menos convencional produce una distensión de las normas y su comprensión más fácil para el receptor. Por ejemplo, muchos de los carteles de aviso japoneses recurren a esta estrategia de comunicación para transmitir un mensaje:

In the Japanese culture, the cute aesthetic is widely used by many organizations and for many purposes including mascots for the police, warning signs for dangerous areas, pedestrian detours in public places, company mascots, and video game characters, among others. (Cheok y Fernando 2012: 299)

Además, esto se demuestra en las comunicaciones a diario. Cuando los interlocutores tratan un asunto serio en un ambiente más distendido, están ansiosos por relajar ese nivel de gravedad. Para ello, como indican las investigadoras Katsuno y Yano, utilizan tantos los emoji como los kaomoji ${ }^{4}$ para suavizarlo (2002: 215).

La segunda función es una mejor asimilación del contenido. Piscólogos japoneses descubrieron que el mismo ejercicio con un personaje kawaii permitía una mayor concentración en las tareas a realizar y ayudaban a recordar mejor los ejercicios, en contraste con la prueba sin dicho componente (Nittono et al. 2012).

Desde el punto de vista teórico, lo kawaii es un recurso sensual muy útil por su apariencia inofensiva y eficacia en la comunicación. Por lo tanto, no es extraño que se encuentre en infinidad de productos comerciales, tanto en tierras niponas como extranjeras.

\section{Squishy Tank}

Squishy Tank es un videojuego, para la Nintendo DS, basado en Yawaraka Sensha (Tanque blando). Este personaje apareció en los años 2000 en una serie de animación por flash que relata sus desventuras en la guerra. Su imagen recuerda vagamente a un tanque y aparecen algunas de las características de lo kawaii: un color plano predominante, ojos grandes, extremidades pequeñas en forma de orugas de tanque, cuerpo con redondo... Su nombre en japonés está compuesto por la palabra yawaraka (blando);

${ }^{4}$ Un kaomoji es una variación de los emoji que están más relacionados con la estética japonesa actual. Con una serie de caracteres, representan caras y emociones como alegría $\left\langle{ }^{\wedge} \mathrm{o}^{\wedge}\right\rangle$, acciones como escuchar música $« \mathrm{~d}-\_-b », \mathrm{o}$ incluso animales como el gato $«=\wedge . \wedge=»$. 
así cumple con la realidad táctil que incorpora la palabra kawaii. Incluso hace referencia a la cualidad sonora: se le asocia con la palabra moco moco, un giatigo ${ }^{5}$ característico de los objetos redondos que se pueden apretar y recuperar su estado original.

En este juego no es un FPS, sino un juego de puzles. Los FPS -o First Person Shooter- son el género predilecto de los juegos con contenido bélico, junto con los de estrategia; se fundamentan por su punto de vista en primera persona y su alto contenido violento. Algunos ejemplos son los juegos como Wolfestein 3D (1992), de id Software, o Soldier of Fortune (2000), de Raven Software. En este caso, las mecánicas del puzle hacen el juego más atractivo para el público mayoritario. Para añadir, los videojuegos en primera persona no son del agrado del consumidor japonés.

El objetivo del juego es conseguir eliminar el mayor número de casillas posibles para que no eliminen los tanques blandos. Si el jugador falla, una explosión ocurre y desaparecen la mayoría. Con esta mecánica, junto con los gráficos, se desentraña el siguiente mensaje: la guerra es necesaria porque protege a los más débiles de las amenazas externas.

Por consiguiente, este juego, tanto por su estética como por sus mecánicas, transmite la necesidad de proteger al más débil. Por último, esta proclama puede llegar a todas los estratos de los consumidores por su aspecto y su género.

\section{El moe y su atracción}

La otra corriente estética a estudiar corresponde al moe. Esta tendencia estilística comenzó en los años 90, aunque el origen no es concreto. Una de las posibles procedencias corresponde a la adoración del personaje Sagisawa Moe de la serie de animación Kyōryū Wakusei (Planeta dinosaurio), emitida entre los años 1993 y 1994 (Saitō 2011: 129). Otro posible origen al término corresponde a parte de la reacciones de los fans de Tomoe Hotaru, en el doblaje castellano como Andrea, de Sailor Moon: Hotaru ni moe moe (Saitō 2011: 29).

En la realidad visual, el moe comparte ciertos rasgos con el kawaii. Algunos de estos rasgos son las chicas jóvenes, los ojos grandes, cierto grado de indefensión y delicadeza, cuerpos estilizados... De cualquier modo, su componente central es la fijación por un sujeto de ficción y el contexto en el que se mueve. El desarrollo de una manifestación moe se centra más en las características sensoriales en los personajes que un desarrollo profundo de la historia o de un mensaje. Hiroki Azuma resume de esta manera el concepto de moe: «L'absence de récit ou de message est comme remplacé par un grand déploiement de techniques visant à l'augmentation de la force d'attraction sensuelle du personnage» (2008: 73).

Lo moe recurre a una identificación extrema que roza el fetichismo. Lo importante es el personaje, y en menor grado su personalidad, porque, en la posmodernidad, ya no existe ni una historia clara ni un mensaje interesante:

\footnotetext{
${ }^{5} \mathrm{O}$ sonido mimético Es un tipo de onomatopeya que se asocia a acciones, estados y formas que no producen sonido. Otro ejemplo es nico nico que se traduce por «sonreír».
} 
En conséquence ce qui constitue le lien entre tous les développements, ce n'est ni la personnalité de l'auteur, ni le message, mais un univers ou des personnages communs, voire, dans des cas extrêmes, uniquement des personnages ans aucune référence à un contexte. (Azuma 2008: 74)

En relación con el pensamiento de François Lyotard, este fetichismo es una clara muestra de cómo los grand-recit están cayendo y los seguidores solo buscan consumir los protagonistas de la cultura popular. Entonces, los consumidores no se preocupan por el supuesto mensaje que pueden contener un videojuego bajo el aspecto visual del moe; pero esto no significa que esté ausente.

\section{Valkyria Chronicles}

El videojuego Valkyria Chronicles es el modelo de juego con personajes moe. Narra la historia de la defensa del país Galia ante la invasión de la Alianza Imperial. El protagonista principal es Welkin Gunther, hijo del General Belgen Gunther, que, junto a sus amigos, luchará por su vida y la de su familia. Se unirá como integrante de la milicia en la recién formada Squad 7 cuyo objetivo es repeler la invasión y descubrir los verdaderos propósitos del ataque.

Además de su estética moe, se añade las influencias del periodo de entreguerras y de la Segunda Guerra Mundial. Por ejemplo, el ejército de la Alianza Imperial recuerda a la Alemania del III Reich, con toques de la Rusia zarista. El uso de la imaginería nazi para identificar al enemigo no es casual: Galia es un pequeño país que insinúa ser Bélgica u Holanda, países que sufrieron la invasión y ocupación alemana. Como es lógico, su uso refuerza el papel del enemigo.

Por último, el estilo del cómic japonés aparece en el juego. Uno de estos elementos son las onomatopeyas. Cuando ocurre un efecto sonoro en las escenas de combate, surge una onomatopeya para enfatizarlo. Otro elemento común a los mangas es el uso de colores pastel, muy popular entre dibujantes y artistas. Todo el aparato gráfico del juego está organizado para que la experiencia sea más parecida a un manga que a un videojuego bélico estándar.

En cuanto al género, es un RPG -o juego de rol- con toques de estrategia. La elección de este género no es baladí porque es el género de videojuegos más popular entre los japoneses y permite desarrollar un apego especial por los personajes. Una de las mecánicas especiales de este juego son los movimientos especiales con efectos beneficios para el jugador. También destaca la ausencia de sangre. Todos estos elementos hacen que la experiencia del juego sea irreal y no tenga nada que ver con la guerra en sí. Esta suavización ocurre porque facilita la recepción del siguiente mensaje.

Cuando Welkin Gunther tiene que luchar, lo hace con el convencimiento de que debe acabar con la invasión enemiga y así poder vivir en paz con su familia. Esto, que según Hiroki Azuma no existe ni se transmite; llega a los consumidores gracias a los fanes. El desarrollo de la cultura fan en Japón es importante y genera en distintos productos transmedia, como pueden ser los fanzines - o dōjinshi- o el cosplay. 
Estas manifestaciones lo perpetúan, sobre todo en el caso del cosplay. El cosplay no es solo disfrazarse de un personaje, sino actuar igual que él y reproducir los mismos mensajes. Así, de este modo, se difunde a modo de altavoz entre aficionados y consumidores, aunque no sea de forma consciente.

Como en el juego Squishy Tank, reaparece el mismo contenido: proteger al débil. Así, se puede decir que la estrategia de suavización de los dos videojuegos hace que la visión de la guerra no sea, por lo menos, negativa y justifica la defensa del modus vivendi por parte del ejército.

\section{Conclusiones}

Esta investigación ha analizado la estética y las mecánicas de dos videojuegos japoneses. La primera conclusión que aparece es que las retóricas de comunicación en los videojuegos japoneses se fundamentan principalmente en lo estético para suavizar el mensaje probélico.

La segunda conclusión es que los videojuegos japoneses promocionan una imagen positiva del ejército y las guerras. Este no se fundamenta en la superioridad racial, como en la Segunda Guerra Mundial, sino en la defensa y protección de los individuos más indefensos.

La tercera conclusión es que esta propaganda probélico no solo se encuentra en lo estético. En los dos juegos analizados, se abogan por utilizar géneros alejados a los juegos típicos asociados a lo bélico como los FPS o la estrategia. Ambos utilizan un género más accesible al público en general, como el caso del puzle, o el más popular entre los jugadores japoneses, el RPG.

Ante estas conclusiones, se afirma que en una sociedad donde se fomenta una cultura pacifista, pueden existir este tipo de expresiones culturales. Sin embargo, esta investigación no ha analizado un punto de interés sobre este fenómeno: ¿quién fomenta este mensaje? Para responderla, es conveniente crear futuras líneas al respecto.

Por último, es interesante ampliar el estudio de los videojuegos en los estudios sobre propaganda. Es un campo fértil dentro de los game studies, pero no en el resto de disciplinas. Tanto por su actualidad como por sus características diferenciadoras, son de gran interés para estudiar su relación con las distintas ramas del conocimiento.

\section{Referencias bibliográficas}

AzuMA, Hiroki (2008): Génération Otaku: Les enfants de la postmodernité. Paris: Hachette littérature.

Bogost, Ian (2010): Persuasive Games: The Expressive Power of Videogames. London, Cambridge (U.S.A): The MIT Press.

CAILloIs, Roger (1976): Les jeux et les hommes. Paris, Gallimard.

CHeOK, A. D., y Fernando, O. N. N. (2012): «Kawaii/Cute interactive media». Universal Access in the Information Society, 11(3): 295-309. <http://dx.doi.org/10.1007/s10209011-0249-5> 
Crogan, Patrick (2011): Gameplay Mode: War, Simulation, and Technoculture. Minneapolis, London: University of Minnesota Press. <http://dx.doi.org/10.5749/minnesota/9780816653348.001.0001>

Dunningan, James F. (2016): «Foreword: The Paper Time Machine goes Electric», en Zones of Control: Perspectives on Wargaming, Pat Harrian y Matthew Kirschenbaum (eds.), pp. XXXIII-XXXVIII. London, Cambridge (U.S.A): The MIT Press.

Gould, Stephen J. (2008): «A Biological Homage to Mickey Mouse». Ecotone, 4(1): 333340. <http://dx.doi.org/10.1353/ect.2008.0045>

Huizinga, Johan (2010): Homo ludens. Madrid, Alianza.

KATSUNO, Hirofumi, y YANO, Christine R. (2002): «Face to Face: On-Line Subjectivity in Contemporary Japan». Asian Studies Review, 26(2): 205-231. $<$ http://dx.doi.org/10.1080/10357820208713341>

Kawato, Yuko, PekKanen, Robert J. y Yamamoto, Hidehiro (2016): «State and Civil Society in Japan», en The Routledge Handbook of Japanese Politics, Alisa Gaunder (ed.), pp. 117-129. London, New York: Routledge. <http://dx.doi.org/10.4324/ 9780203829875.ch11>

KINNEY, Jack (dir.) (1943): Der Fuehrer's Face. EE.UU.: Walt Disney Productions.

KinSELlA, Sharon (1995): «Cuties in Japan», en Women, Media and Consumption in Japan, Lise Skov y Brian Moeran (eds.), pp. 220-254. London: Curzon Press.

KoBAYASHI, Masaki (dir.) (1959): Ningen no jōken (La condición humana). Japón: Masaki Kobayashi, Watatsuki Shigeru.

KogawA, Asami (dir.) (1993-1994): Kyōryū Wakusei. Japón: NHK, Studio Junio.

MiLIUS, John (dir.) (1984): Red Dawn (Amanecer rojo). EE.UU.: Valkyrie Films, United Artists.

MorREALl, James (1991): «Cuteness». British Journal of Aesthetics, 31(1): 39-47. $<$ http://dx.doi.org/10.1093/bjaesthetics/31.1.39>

MurAKAMI, Haruki (2006): Crónica del pájaro que da cuerda al mundo. Barcelona: Tusquets.

NAKAZAWA, Keiji (2015-2016): Pies descalzos: Una historia de Hiroshima. Barcelona: Debolsillo.

NitTono, Hiroshi, Fukushima, Michiko, Yano, Akihiro y MoriYA, Hiroki (2012): «The Power of Kawaii: Viewing Cute Images Promotes a Careful Behavior and Narrows Attentional Focus». PLOS ONE 7(9). En: http://journals.plos.org/plosone/article? $\mathrm{id}=10.1371$ /journal.pone.0046362. [Consulta: 05/04/2016] <http://dx.doi.org/10.1371/ journal.pone.0046362>

SAITō, Tamaki (2011): Beautiful Fighting Girl. Minneapolis, London: University of Minnesota Press. <http://dx.doi.org/10.5749/minnesota/9780816654505.001.0001>

TAKEUCHI, Naoko (cr.) (1992-1997): Bishōjo Senshi Sailor Moon (Sailor Moon). Japón: Asahi TV, Tōei Agency, Tōei Dōga.

TATE, Trudi (2009): «The First World War: British writing», en The Cambridge Companion to War Writing, Kate McLoughlin (ed.), pp. 160-175. Cambridge, New York: Cambridge University Press. <http://dx.doi.org/10.1017/ccol9780521895682.015>

YAMOTA, Inuhiko (2015): Kawaiiron [Teoría de lo adorable]. Tōkyō: Chikuma Shobō.

\section{Ludografía}

CAPCOM (1991): Street Fighter II: The World Warrior. (Recreativa). 
Central Internet Bureau (2007): Special Force 2: Tale of the Truthful Pledge. (PC): Hezbollah.

GAME FreaK, Creatures (1996): Pokémon. (Game Boy): Nintendo.

ID SOFTWARE (1993): Wolfestein 3D. (PC): GT Interactive

PETA (2008): Cooking Mama, The Unauthorized Peta Edition: Mama kills animals. (Flash)

RAVEN SOFTWARE (2000): Soldier of Fortune. (PC): Activision.

SEGA (2008): Valkyria Chronicles. (Playstation 3).

SuCCESS CORP (2010): Squishy Tank. (Nintendo DS): Natsume.

THE InTERNATIONAL CONSORTIUM OF InVESTIGATIVE JOURNALISTS (2016): Starway to Tax Heavens. (Flash)

United STATES ARMY (2002): America's Army. (PC).

VALVE (1999): Counter-Strike. (PC) 